\title{
Mutant-allele tumor heterogeneity in malignant glioma effectively predicts neoplastic recurrence
}

\author{
PENGFEI WU, WEI YANG, JIANXING MA, JINGYU ZHANG, MAOJUN LIAO, \\ LUNSHAN XU, MINHUI XU and LIANG YI
}

Department of Neurosurgery, Daping Hospital and Institute Research of Surgery, Army Medical University, Chongqing 400042, P.R. China

Received March 13, 2019; Accepted September 6, 2019

DOI: $10.3892 / 01.2019 .10978$

\begin{abstract}
Intra-tumor heterogeneity (ITH) is one of the most important causes of therapy resistance, which eventually leads to the poor outcomes observed in patients with glioma. Mutant-allele tumor heterogeneity (MATH) values are based on whole-exon sequencing and precisely reflect genetic ITH. However, the significance of MATH values in predicting glioma recurrence remains unclear. Information of patients with glioma was obtained from The Cancer Genome Atlas database. The present study calculated the MATH value for each patient, analyzed the distributions of MATH values in different subtypes and investigated the rates of clinical recurrence in patients with different MATH values. Gene enrichment and Cox regression analyses were performed to determine which factors influenced recurrence. A nomogram table was established to predict 1-, 2- and 5-year recurrence probabilities. MATH values were increased in patients with glioma with the wild-type isocitrate dehydrogenase $\left(\mathrm{NADP}^{(+)}\right)$ (IDH)1/2 (IDH-wt) gene $(\mathrm{P}=0.001)$ and glioblastoma (GBM; $\mathrm{P}=0.001)$. MATH values were negatively associated with the 2- and 5-year recurrence-free survival (RFS) rates in patients with glioma, particularly in the IDH1/2-wt and GBM cohorts ( $\mathrm{P}=0.001$ and $\mathrm{P}=0.017$, respectively). Furthermore, glioma cases with different MATH levels had distinct patterns of gene mutation frequencies and gene expression enrichment. Finally, a nomogram table that contained MATH values could be used to accurately predict the probabilities of the 1-, 2- and 5-year
\end{abstract}

Correspondence to: Professor Liang Yi or Professor Minhui $\mathrm{Xu}$, Department of Neurosurgery, Daping Hospital and Institute Research of Surgery, Army Medical University, 10 Changjiangzhi Road, Daping, Yuzhong, Chongqing 400042, P.R. China

E-mail: yiliangcq@126.com

E-mail: minhuixu66@aliyun.com

Abbreviations: GBM, glioblastoma; ITH, intra-tumor heterogeneity; LGG, low grade glioma; MATH, mutant-allele tumor heterogeneity; RFS, recurrence-free survival

Key words: glioma, intra-tumor heterogeneity, recurrence, mutant-allele tumor heterogeneity, prediction
RFS of patients with glioma. In conclusion, the MATH value of a patient may be an independent predictor that influences glioma recurrence. The nomogram model presented in the current study was an appropriate method to predict 1-, 2- and 5-year RFS probabilities in patients with glioma.

\section{Introduction}

Glioma is the most common primary malignant tumor in the central nervous system (1). Despite surgery and radiotherapy, chemotherapy and targeted therapy, the majority of malignant gliomas still recur $(2,3)$, which is primarily due to chemo-radiotherapy resistance (4). Previous studies have demonstrated that intra-tumor heterogeneity (ITH) is the main cause underlying resistance to current therapy methods and, ultimately, glioma recurrence (5-8).

ITH was first described by Rudolf Virchow in the 1800s (9), which involved genetic heterogeneity. Subsequently, Peter Nowell put forward the theory of 'Clonal Evolution of Tumor Cell Populations' in 1976 (10). Nowell noted that natural selection gives rise to tumor subclones and that ITH is a basis for tumor evolution (10). With the development of DNA sequencing technology, an increasing number of studies $(11,12)$ support this hypothesis. Several studies have analyzed ITH at multiple loci in tumors or by using single cell sequencing $(13,14)$. However, it would be difficult to implement these methods in large-scale analyses of clinical samples.

Mutant-allele tumor heterogeneity (MATH) values are indicators of gene mutation dispersion that were developed by Mroz and Rocco (15). These values are based on whole-exome sequencing (WES) of tumor tissues. Sub-clonal mutations and copy-number aberrations can influence MATH values (15). Importantly, ITH can be quantified by comparing the fraction of tumor gene mutations to that of normal gene mutations, as has been reported in head and neck (16), rectal (17), breast (18), and lung cancer (19).

The present study calculated MATH values of low grade glioma (LGG) and glioblastoma (GBM) samples using data from The Cancer Genome Atlas (TCGA) database. Subsequently, the distributions of MATH values in different glioma subtypes were analyzed. Furthermore, the results of gene expression enrichment analysis in cohorts with different MATH levels were reported. Finally, the association between 
MATH levels and glioma recurrence was verified, and a nomogram table was built to predict the probabilities of glioma recurrence-free survival (RFS) time.

\section{Materials and methods}

Patients and clinical variables. The whole data (LGG and GBM; obtained from 41 clinical centers) used in the present study were downloaded from the TCGA database (https://portal.gdc.cancer.gov; project ID: TCGA-LGG and TCGA-GBM, downloaded on April 3, 2018). Clinical data were obtained for 515 patients with LGG and 596 patients with GBM. WES data were obtained for 508 patients with LGG and 390 patients with GBM. RNA sequencing data were obtained for 529 patients with LGG and 174 patients with GBM. Cases of recurrent status with uncertainty, contradiction or lack of clinical data were excluded. Isocitrate dehydrogenase $\left(\mathrm{NADP}^{(+)}\right)(\mathrm{IDH}) 1 / 2$ gene mutation status was obtained from the WES data. Recurrence status, recurrence time, histological type, World Health Organization (WHO) grade (2007 WHO classification) and clinical information were obtained from the clinical follow-up data.

Generation of MATH values. MATH values were calculated according to the method described by Mroz and $\operatorname{Rocco}(15,16)$. The 't_ref_count' and 't_alt_count' items in the WES data were selected. To obtain the MATH value, the mutant-allele fraction (MAF), median MAF value (mMAF) and median absolute deviation (MAD) were successively calculated to obtain the equation: MATH $=100 * \mathrm{MAD} / \mathrm{mMAF}$. The aforementioned calculations were performed using $\mathrm{R}$ (version 3.5.0); https://www.r-project.org).

Generation of cut-off values. RFS data and MATH values were combined using the Euclidean distance of the receiver operating characteristic (ROC) curve to obtain the cut-off values of the MATH values. The whole patients were separated into highand low-MATH groups. Similarly, the present study obtained the MATH cut-off values in different subtypes including: The IDH-mutant (mut), IDH-wild-type (wt), grade II, grade III, grade IV (GBM), oligodendroglioma, oligo-astrocytoma and astrocytoma groups. Using these cut-off values, the patients were divided into high- and low-MATH groups.

Statistical analysis. The Kolmogorov-Smirnov test (test of normality), unpaired t-test, one-way ANOVA followed by least significant difference post hoc test, $\chi^{2}$ test and Cox regression analysis were performed using SPSS v25.0 (IBM Corp.). Kaplan-Meier analysis and log-rank tests were carried out using STATA v15.0 (StataCorp LP). The nomogram and calibration analyses were performed using $\mathrm{R}$ (version 3.5.0). The discrimination and calibration of the nomogram were validated by discrimination using ROC curve and calibration curve analyses, respectively. FunRich (version 3.1.3; http://funrich.org/download) was used for DNA mutation enrichment analysis of 460 patients with 13,663 mutations in the low-MATH group and 427 patients with 15,174 mutations in the high-MATH group, and gene set enrichment analysis (GSEA; version 3.0; http://software.broadinstitute. org/gsea/index.jsp) was used for RNA enrichment analysis.
The $\chi^{2}$ test was used to compare the DNA mutation rates of patients with different MATH levels. $\mathrm{P}<0.05$ was considered to indicate a statistically significant difference.

\section{Results}

MATH values in different cohorts of patients with glioma. The present study screened a total of 757 patients with complete clinical and total exon sequencing information (Table SI). The obtained MATH values exhibited a normal distribution according to the Kolmogorov-Smirnov test (Fig. 1A). Since the IDH1/2 gene is a vital molecular marker that influences glioma prognosis (20), the present study first investigated the association between MATH values and IDH mutation status. The MATH values were significantly higher in the IDH-wt glioma group than in the IDH-mut glioma group (Fig. 1B). As the MATH value reflected the gene ITH, the results indicated that ITH levels were higher in the IDH-wt group than in the IDH-mut group. Furthermore, MATH values were significantly higher in GBM than in oligodendroglioma and oligo-astrocytoma. However, there was no significant difference observed in MATH values between the GBM and astrocytoma groups ( $\mathrm{P}=0.126$; Fig. $1 \mathrm{C})$. Finally, MATH values were positively associated with glioma WHO grade. MATH values were significantly higher in grade IV glioma than in grade II and III glioma (Fig. 1D). These data indicated that among glioma types, GBM had the highest ITH level.

MATH values and the interval to recurrence are negatively associated.Kaplan-Meier analysis of all patients was performed and suitable cut-off values were utilized to divide the patients with glioma into low- and high-MATH groups. The RFS rates were $43 \%$ at 1 year and $17 \%$ at 2 years in the high-MATH group and 56 and $28 \%$, respectively, in the low-MATH group. Therefore, the RFS rates were higher in the low-MATH group compared with in the high-MATH group $(\mathrm{P}=0.001)$. These results demonstrated that MATH values were negatively associated with the interval to glioma RFS (Fig. 2A).

Patients with glioma with IDH1/2-wt are predicted to have a poor prognosis (20). However, the present study revealed that in the IDH1/2-wt cohort, the interval to neoplastic recurrence was significantly longer in low-MATH patients than in high-MATH patients (Fig. 2B). Similarly, although patients with GBM have been reported to have the shortest interval to recurrence among all patients with malignant glioma (21), the present study revealed that prognoses were significantly improved in patients with GBM with low MATH levels compared with those with high MATH levels (Fig. 2D). In the IDH1/2-mut cohort, glioma MATH levels were not observed to significantly influence the 5-year RFS rate (Fig. 2C).

Gene mutation and expression enrichment in glioma groups with different MATH levels. The patients were divided into low- and high-MATH groups according to the cut-off value shown in Fig. 2A, and then genetic mutations were analyzed in the two groups. The present study revealed that the mutation frequency of single genes distinguished the two groups from each other. It was observed that seven genes [IDH1, tumor protein p53 (TP53), titin (TTN), ATRX chromatin remodeler (ATRX), capicua transcriptional repressor, mucin 16 (MUC16) 

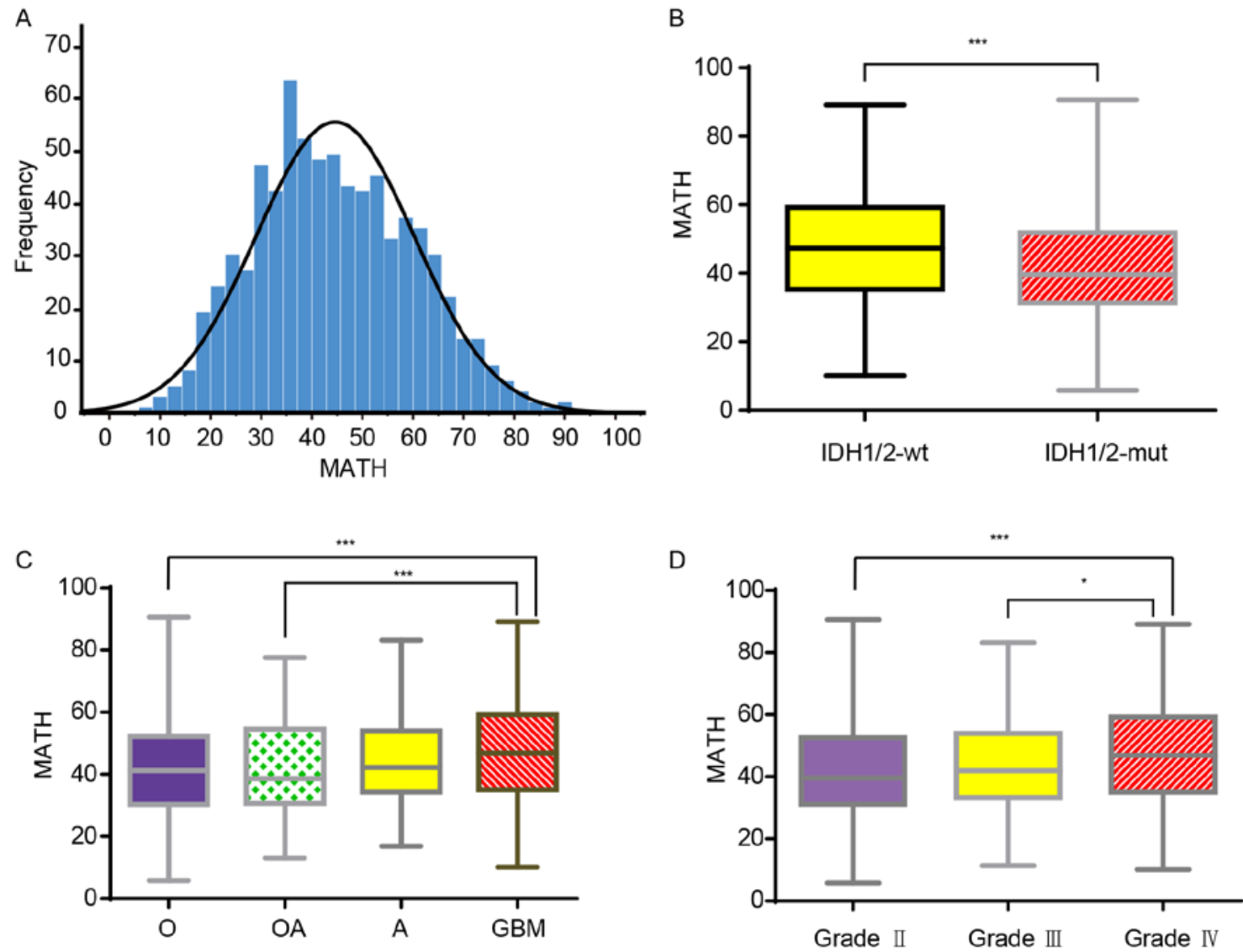

Figure 1. MATH values in different glioma subtypes. (A) Distribution of MATH values among all patients with glioma (LGG and GBM). The MATH values ranged between 5.81 and 90.60, with a mean of 44.61 and a median of 43.28. (B) Boxplot showing the MATH values of patients with different IDH1/2 glioma types. The MATH values found in IDH1/2-wt ranged between 10.12 and 89.15 , with a mean of $47.37 \pm 16.03$. The MATH values found in patients with IDH1/2-mut ranged between 5.81 and 90.60, with a mean of 41.68 \pm 14.70 . (C) Boxplot showing the MATH values found in patients with glioma with different histological classifications. The MATH values in O, OA, A and GBM were 41.39 $\pm 15.17,42.18 \pm 15.26,44.52 \pm 14.56$ and $46.86 \pm 16.13$, respectively, and the ranges were 5.81-90.60, 13.07-77.60, 16.87-83.22 and 10.12-89.15, respectively. (D) Boxplot showing the MATH values obtained for patients with different World Health Organization grades of glioma. The MATH values obtained in grade II, III and IV were $41.77 \pm 15.15,43.62 \pm 14.84$ and $46.86 \pm 16.13$, respectively, and the ranges were $5.81-90.60,11.40-83.22$ and $10.12-89.15$, respectively. Data are presented as the mean $\pm \mathrm{SD} .{ }^{*} \mathrm{P}<0.05,{ }^{* * *} \mathrm{P}<0.001$. A, astrocytoma; GBM, glioblastoma; IDH, isocitrate dehydrogenase $\left(\mathrm{NADP}^{(+)}\right)$; LGG, low grade glioma; MATH, mutant-allele tumor heterogeneity; mt, mutant; O, oligodendroglioma; OA, olio-astrocytoma; wt, wild-type.

and epidermal growth factor receptor (EGFR)] were hypermutated in the low-MATH group (frequency $>10 \%$; Fig. 3A), whereas 13 genes (TTN, TP53, IDH1, MUC16, ATRX, PTEN, EGFR, obscurin cytoskeletal calmodulin and titin-interacting RhoGEF, filaggrin, neurofibromin 1, mucin 17, dynein axonemal heavy chain 3 and LDL receptor related protein 2) were hypermutated in the high-MATH group (Fig. 3B). Therefore, six genes (IDH1, TP53, TTN, ATRX, MUC16 and EGFR) were hypermutated in the low- and high-MATH groups. Using a $\chi^{2}$ test, it was identified that while the difference in TP53 was not significant $(\mathrm{P}=0.91)$, the mutation sample frequencies of IDH1 and ATRX were significantly lower in the high-MATH group, whereas the mutation sample frequencies of TTN, MUC16 and EGFR were significantly higher in the high-MATH group (Fig. 3C).

Furthermore, DNA mutation enrichment analysis demonstrated that enriched and depleted pathways in the high-MATH group were different from those in the low-MATH group (Fig. 3D). Finally, GSEA indicated that there were differences in the expression patterns of the 'cell adhesion molecules cams' (Fig. 3E) and 'cytokine cytokine receptor interaction' signaling pathways (Fig. 3F).
Nomogram based on MATH predicts glioma RFS probability. The present study utilized Cox regression analysis to screen for key factors associated with glioma recurrence. In univariate Cox regression analysis, race and the mutation status of the MUC16 gene had no effect on glioma recurrence. The results of the multivariate Cox regression analysis suggested that MATH level, the mutation status of two genes (IDH and TTN), and four clinical characteristics (age, sex, WHO grade and histological classification) had a significant influence on glioma recurrence. However, the mutation status of the other three genes assessed (TP53, ATRX and EGFR) was not closely associated with recurrence (Table I).

Based on the results of the multivariate Cox regression analysis, the present study developed a nomogram table to predict the probabilities of glioma RFS within 1,2 and 5 years. The total scores shown in the nomogram table reflect the sum of all significant factors, and were positively associated with glioma recurrence (Fig. 4). ROC curve analysis of the nomogram was used to validate the discrimination capability of the nomogram, and the area under the curve values for 1, 2 and 5 years were $0.876,0.903$ and 0.821 , respectively (Fig. 5A-C). Subsequently, a calibration curve was generated to validate 
A

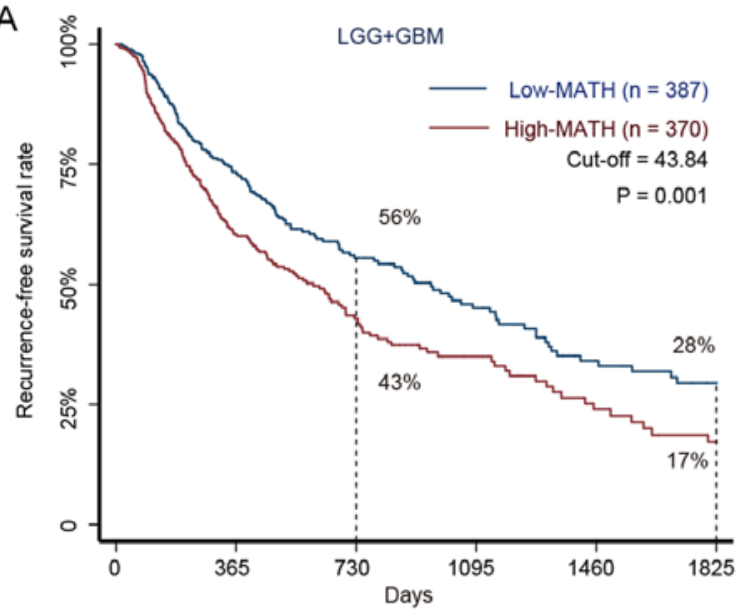

C

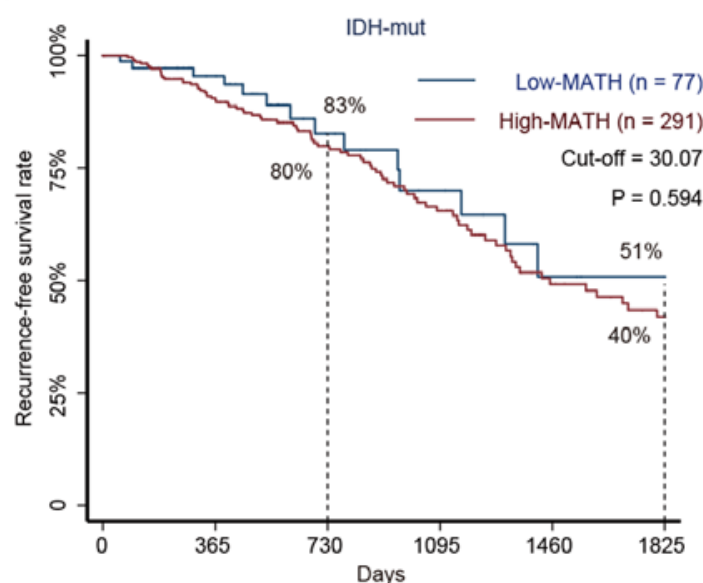

B

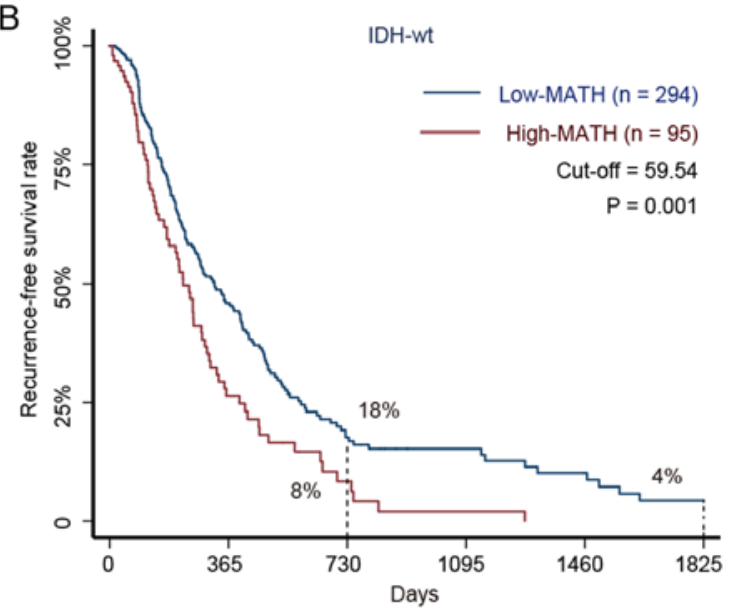

D

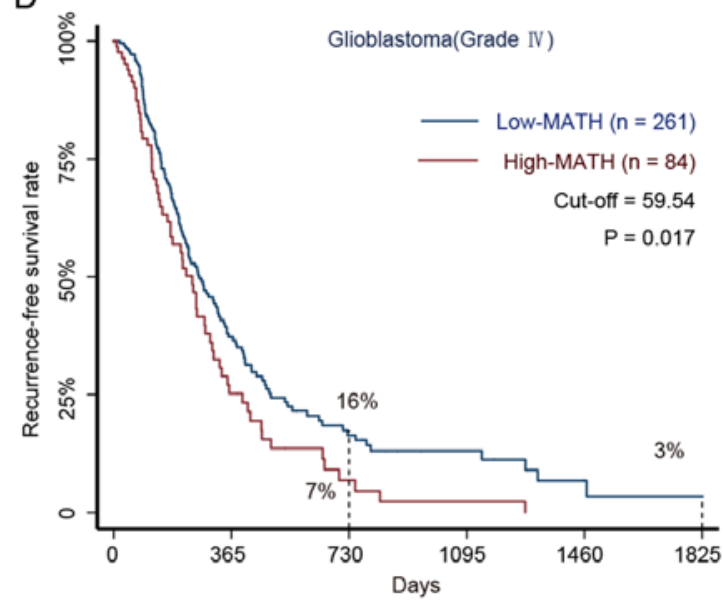

Figure 2. Kaplan-Meier survival curves for patients with glioma with high and low MATH levels. (A) The 5-year RFS rate in 757 patients with glioma (LGG and GBM) with high and low MATH levels. Among patients in the low-MATH group, the RFS rates were $56 \%$ at 2 years and $28 \%$ at 5 years, whereas the rates were 43 and $17 \%$, respectively, in the high-MATH group ( $\mathrm{P}=0.001)$. (B) The 5-year RFS rate in 389 patients with glioma with the IDH-wt subtype and high or low MATH levels. The Kaplan-Meier estimates revealed RFS rates of $18 \%$ at 2 years and $4 \%$ at 5 years in the low-MATH group compared with respective rates of 8 and $<0.001 \%$ in the high-MATH group $(\mathrm{P}=0.001)$. (C) The 5-year RFS rates in 368 patients with the IDH-mut subtype and high or low MATH levels. Patients in the low-MATH group had an RFS rate of $83 \%$ at 2 years and $51 \%$ at 5 years, whereas the corresponding rates were 80 and $40 \%$ in the high-MATH group (P=0.594). (D) The 5-year RFS rates in 345 patients with GBM with high or low MATH levels. Patients in the low-MATH group had RFS rates of $16 \%$ at 2 years and 3\% at 5 years, whereas those in the high-MATH group had rates of 7 and $<0.1 \%$, respectively ( $\mathrm{P}=0.017$ ). The log-rank test was used to determine significance. GBM, glioblastoma; IDH, isocitrate dehydrogenase $\left(\mathrm{NADP}^{(+)}\right)$; LGG, low grade glioma; $\mathrm{MATH}$, mutant-allele tumor heterogeneity; mut, mutant; RFS, recurrence-free survival; wt, wild-type

the calibration of the nomogram table (Fig. 5D-F). The trends indicated by the curve were consistent with the actual RFS probability curves at 1,2 and 5 years. Since the patients came from multiple clinical centers, there was no need for external validation (22). In addition, discrimination and calibration analyses revealed good correspondence between the predicted probabilities of RFS and the actual probabilities within 1, 2 and 5 years. In summary, the nomogram presented in the current study effectively predicted glioma RFS probabilities.

\section{Discussion}

The present study combined LGG and GBM data, and compared the genetic difference between different MATH levels of glioma. Firstly, combining LGG and GBM data could help screen efficient predictors more accurately. LGG data from TCGA database contains grade II and III glioma, which has malignant progression. In addition, the majority of LGG cases may eventually progress to GBM, which is combined with continuous evolution of gene expression (23). Therefore, gene expression of each grade of glioma is closely associated, and it is valuable to analyze the genetic heterogeneity of glioma throughout the whole evolution process.

The analysis confirmed that ITH is an indicator of poor prognosis in glioma. Patients with GBM and IDH1/2-wt glioma had higher MATH levels than the other patients with glioma. When analyzing mutation genes, both the single gene mutations and signaling pathways reflected by the mutant gene sets were different between the high- and low-MATH groups. In addition, the gene expression enriched signaling pathways differed between the high- and low-MATH groups. Furthermore, MATH values were independent predictors of glioma recurrence $(\mathrm{P}=0.015)$. However, histological typing cannot accurately predict glioma prognosis, since a number of patients with LGG had an even worse prognosis than those with $\operatorname{GBM}(24,25)$. Additionally, $\sim 15 \%$ of patients with GBM have good prognosis, and $>2 \%$ of patients with GBM achieve long term survival ( $>10$ years) (26). In certain cohorts 
A

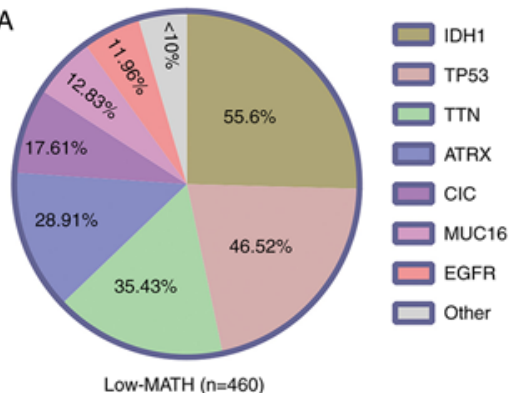

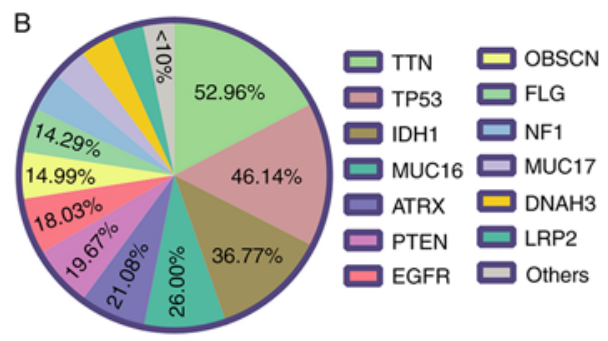

High-MATH $(n=427)$
IDH1 TP53 TTN ATRX MUC16 EGFR $P \leq 0.01 \quad P=0.91 \quad P \leq 0.01 \quad P \leq 0.01 \quad P \leq 0.01 \quad P=0.01$

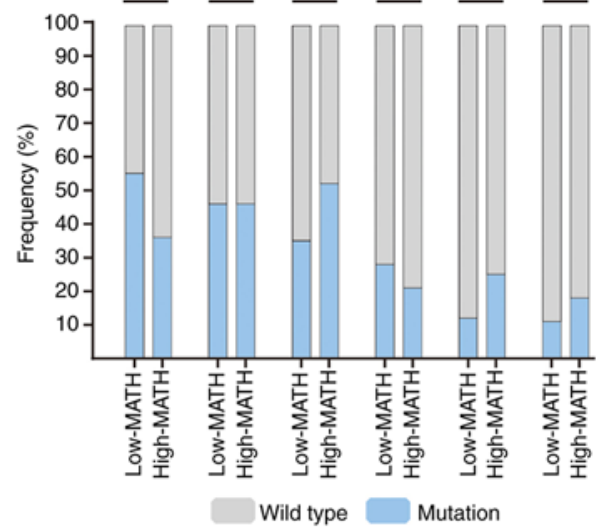

D



$\mathrm{E}$

KEGG: Cell adhesion molecules cams

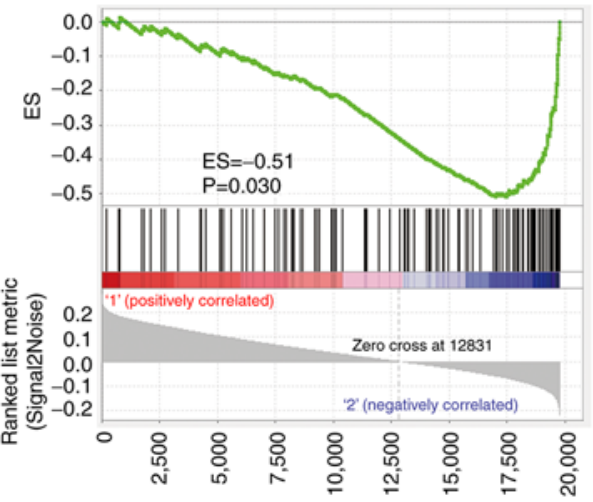

F KEGG: Cytokine cytokine receptor interaction

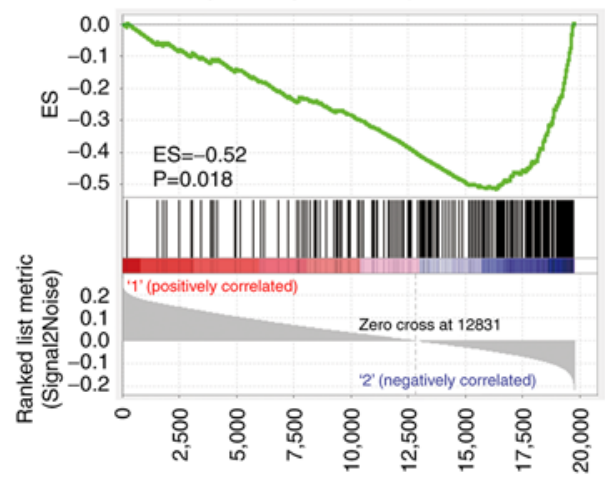

Figure 3. Somatic mutation sample frequency pattern analysis and gene set enrichment analysis. (A) Mutated genes with higher sample frequency in the low-MATH group of patients. (B) Mutated genes with higher sample frequency in the high-MATH group of patients. (C) Comparison of six common high sample frequency mutation genes (IDH1, TP53, TTN, ATRX, MUC16 and EGFR) at different levels in the MATH group. Comparisons were made using the $\chi^{2}$ test. (D) Mutated genes enriched in biological pathways with fold-changes $>100$. Compared with low-MATH glioma, high-MATH glioma exhibited more gene mutations in the $\mathrm{BH} 3$ anti-apoptotic signaling pathway and fewer in the dolichyl phosphate biosynthesis signaling pathway. The gene expression set observed in the high-MATH group was enriched in (E) 'cell adhesion molecules cams' $(\mathrm{P}=0.030$; FDR $\mathrm{q}<0.25)$ and (F) 'cytokine cytokine receptor interaction' signaling pathways $(\mathrm{P}=0.018$; FDR $\mathrm{q}<0.25)$. ATRX, ATRX chromatin remodeler; $\mathrm{CIC}$, capicua transcriptional repressor; DNAH3, dynein axonemal heavy chain 3; EGFR, epidermal growth factor receptor; ES, enrichment score; FDR, false discovery rate; FLG, filaggrin; IDH1, isocitrate dehydrogenase $\left(\right.$ NADP $\left.^{(+)}\right)$ 1; KEGG, Kyoto Encyclopedia of Genes and Genomes; LRP2, LDL receptor related protein 2; MATH, mutant-allele tumor heterogeneity; MUC16, mucin 16; MUC17, mucin 17; NF1, neurofibromin 1; OBSCN, obscurin, cytoskeletal calmodulin and titin-interacting RhoGEF; TP53, tumor protein p53; TTN, titin.

predicted to have a poor prognosis, including the IDH-wt and GBM groups, MATH levels distinguished patients with a relatively good prognosis. Finally, the present study established a nomogram model based on MATH values that accurately predicted glioma recurrence. The present study integrated the factors and accurately predicted the prognosis of glioma, provided a novel idea for the classification of glioma and increased the understanding of malignant glioma in terms of gene heterogeneity.
The effect of ITH on treatment resistance and the results of the gene enrichment analysis may explain the poor outcomes observed in patients with glioma with high MATH levels. High ITH levels have been found to increase chemoradiotherapy resistance in glioma (27). In the present study, the results of the analyses of gene mutation patterns and signaling pathway enrichment were much more complicated in the high-MATH group than in the low-MATH group. Glioma progression conforms to Darwinian theory in that tumors with 
Points

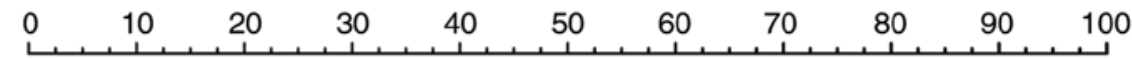

Age

$\stackrel{\text { 45 65y }}{\stackrel{1}{<45 y}>65 y}$

Sex



Female

Histological classification

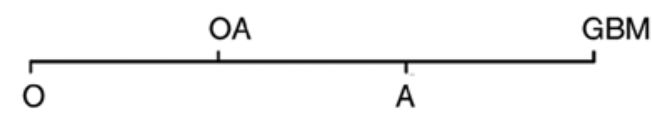

WHO grade

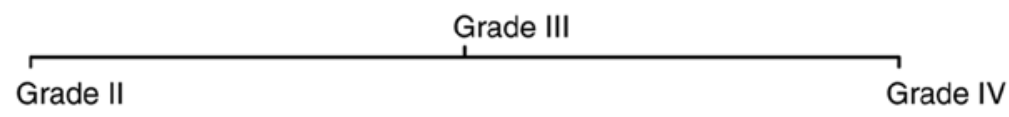

MATH value

$\overbrace{525}^{25 \sim 50}>75$

IDH1/2

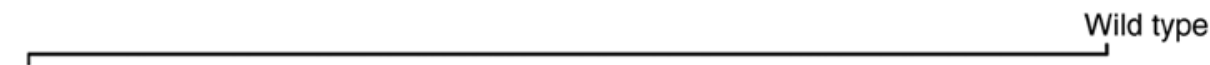

Mutated type

TTN

Wild type

Mutated type

Total points

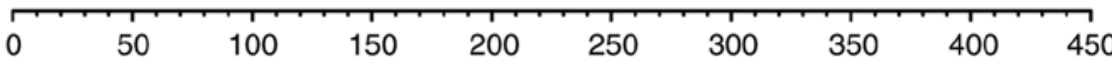

1-year RFS probability



2-year RFS probability

$$
0.9
$$

0.8

0.70 .6

(1)

5-year RFS probability

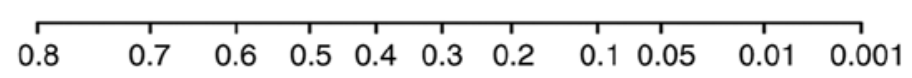

Figure 4. Nomogram prediction table for glioma RFS probability. Each patient can be assigned a point value from the point scale for each factor. The cumulative number of points are identified on the total points bar and used to determine the 1-, 2- and 5-year RFS probabilities. A, astrocytoma; IDH, isocitrate dehydrogenase $\left(\mathrm{NADP}^{(+)}\right)$; MATH, mutant-allele tumor heterogeneity; O, oligodendroglioma; OA, oligo-astrocytoma; RFS, recurrence-free survival; TTN, titin; WHO, World Health Organization.

a high ITH level possess more tumor subclones $(28,29)$, which is beneficial for coping with external selective pressure. In patients receiving chemotherapy drugs or radiation, high ITH gliomas will have more residual subclones to replace those that are lost $(30,31)$. Therefore, compared with the low-MATH group, the high-MATH group tended to have a shorter interval to recurrence. Additionally, gene enrichment analysis demonstrated that the gene mutations occurring in patients with high MATH values were enriched in the 'BH3 anti-apoptotic', 'MAD2 inhibitory signal' and 'glutathione biosynthesis' signaling pathways, which act to inhibit apoptosis (32), mitotic catastrophe and radio-chemotherapy resistance (33), respectively (Fig. 3D). In addition, gene expression was enriched in 'adhesion molecules' and 'cytokine interaction' signaling pathways (Fig. 3E and F). These two signaling pathways act on the tumor microenvironment and provide a driving force for glioma invasion, migration and growth (34). Therefore, patients with high MATH values have a genetic and expression background that indicates a worse prognosis, which is consistent with the results of the survival analysis. 
Table I. Univariate and multivariate Cox analyses for factors with a significant influence on glioma recurrence.

\begin{tabular}{|c|c|c|c|c|c|c|}
\hline \multirow[b]{2}{*}{ Clinical and molecular factors $(\mathrm{n}=757)$} & \multicolumn{3}{|c|}{ Univariate analysis } & \multicolumn{3}{|c|}{ Multivariate analysis } \\
\hline & HR & $95 \% \mathrm{CI}$ & P-value & HR & $95 \% \mathrm{CI}$ & P-value \\
\hline $\begin{array}{l}\text { Age }(18-45 \text { years }=1,46-65 \text { years }=2, \\
>65 \text { years }=3)\end{array}$ & 2.270 & $1.951-2.641$ & $<0.001$ & 1.011 & $1.508-1.547$ & 0.011 \\
\hline Sex $($ male $=1$, female $=0)$ & 1.354 & $1.902-1.679$ & 0.006 & 0.705 & $0.571-0.888$ & 0.003 \\
\hline $\begin{array}{l}\text { Ethnicity }(\text { Asian }=1, \text { Black or } \\
\text { African American=2, White }=3 \text {, Other }=4 \text { ) }\end{array}$ & 1.050 & $0.919-1.198$ & 0.474 & l & 1 & I \\
\hline MATH $(0-25=1,25-50=2,50-75=3,75-100=4)$ & 1.307 & $1.123-1.521$ & 0.001 & 1.198 & $1.037-1.410$ & 0.015 \\
\hline WHO grade $(\mathrm{G} 2=2, \mathrm{G} 3=3, \mathrm{GBM}=4)$ & 3.081 & $2.632-3.607$ & $<0.001$ & 1.478 & $1.118-1.976$ & 0.006 \\
\hline $\begin{array}{l}\text { Histological classification (Oligodendroglioma }=1 \text {, } \\
\text { Oligoastrocytoma }=2 \text {, Astrocytoma }=3, G B M=4)\end{array}$ & 2.107 & $1.876-2.367$ & $<0.001$ & 1.214 & $1.012-1.473$ & 0.037 \\
\hline IDH1/2 $($ wild type $=0$, mutated type $=1)$ & 0.172 & $0.136-0.217$ & $<0.001$ & 0.364 & $0.261-0.561$ & $<0.001$ \\
\hline TTN (wild type $=0$, mutated type $=1$ ) & 1.340 & $1.050-1.697$ & 0.018 & 0.782 & $0.599-0.992$ & 0.043 \\
\hline MUC16 (wild type $=0$, mutated type $=1$ ) & 1.010 & $0.724-1.402$ & 0.965 & l & I & 1 \\
\hline TP53 $($ wild type $=0$, mutated type $=1$ ) & 0.622 & $0.501-0.772$ & $<0.001$ & 0.792 & $0.607-1.011$ & 0.061 \\
\hline ATRX (wild type $=0$, mutated type $=1$ ) & 0.499 & $0.382-0.652$ & $<0.001$ & 1.171 & $0.802-1.561$ & 0.508 \\
\hline EGFR (wild type $=0$, mutated type $=1$ ) & 2.130 & $1.601-2.834$ & $<0.001$ & 0.949 & $0.686-1.254$ & 0.625 \\
\hline
\end{tabular}

TTN, titin; MUC16, mucin 16; TP53, tumor protein p53; ATRX, ATRX chromatin remodeler; EGFR, epidermal growth factor receptor; GBM, glioblastoma; HR, hazard ratio; IDH, isocitrate dehydrogenase $\left(\mathrm{NADP}^{(+)}\right)$; MATH, mutant-allele tumor heterogeneity; WHO, World Health Organization.
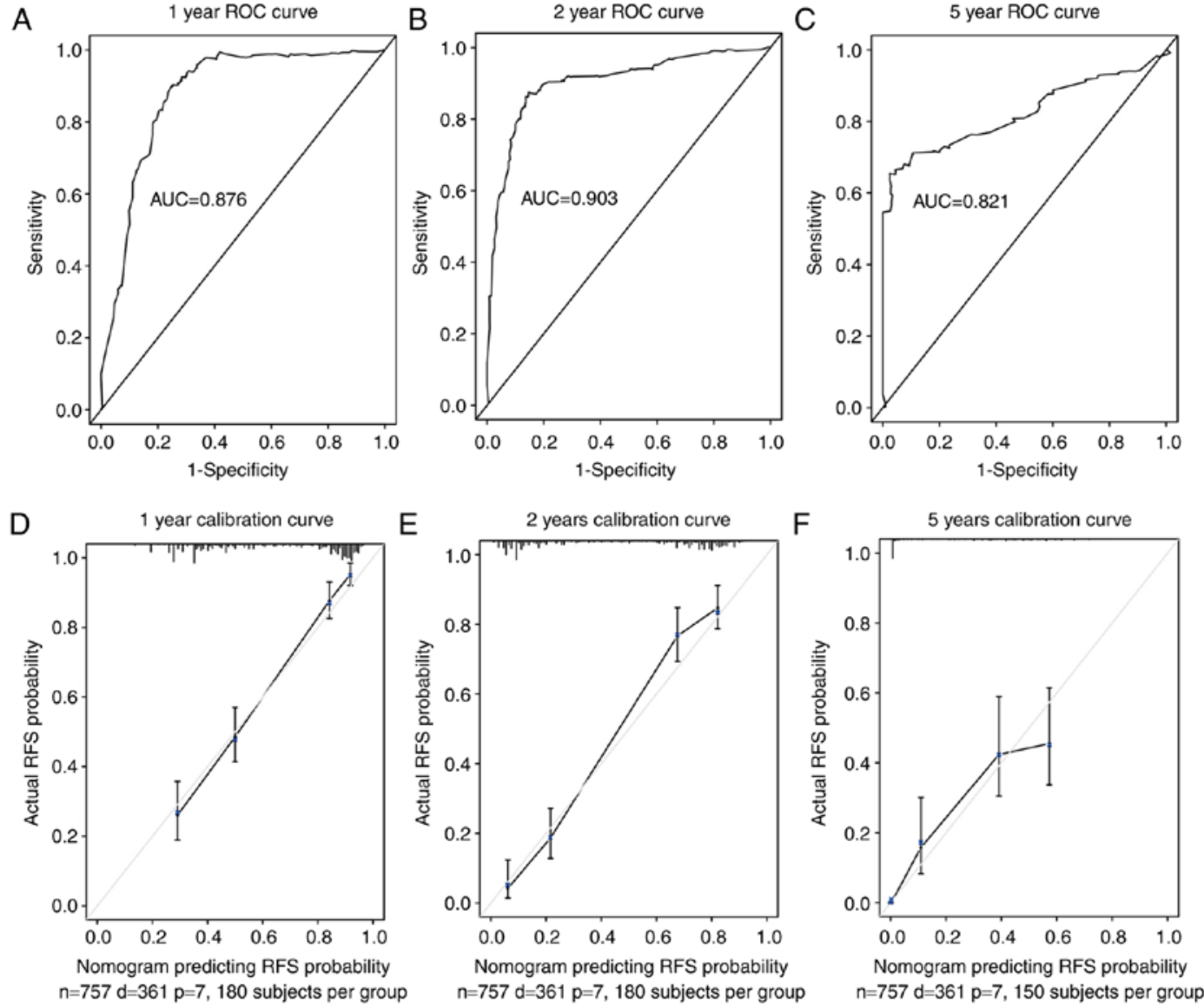

Figure 5. Internal validation of the nomogram designed to predict the probability of glioma RFS at 1,2 and 5 years. ROC curves of the nomogram to predict the probability of glioma RFS at (A) 1, (B) 2 and (C) 5 years. Calibration curve for predicting the probability of RFS at (D) 1 , (E) 2 and (F) 5 years. The grey line indicates the ideal nomogram RFS line. The vertical bars represent the $95 \%$ CI. AUC, area under the curve; RFS, recurrence-free survival; ROC, receiver operating characteristic. 
In the nomogram, several factors including, MATH level, gene IDH1/2, gene TTN, age, sex, WHO grade and histological classification, were indicated to have a substantial effect on glioma recurrence. Age has been identified as an independent prognostic factor in high-grade glioma (35), and elderly patients with glioma exhibit abnormal repair functions, resulting in gene mutations and impaired DNA metabolic functions. Therefore, compared with young patients with glioma, older patients are more likely to have higher tumor ITH levels. In addition, elderly patients tend to choose palliative surgery and low-dose chemo-radiotherapy treatment to avoid severe complications (36), and residual glioma cells can resist chemoradiotherapy by increasing their DNA mutation rate, which may also increase the ITH level in glioma (30). Furthermore, prognoses in glioma are worse in female patients than in male patients (37). However, in the present study, ITH levels were not significantly different between male and female patients with glioma (data not shown). Additionally, while IDH1/2 mutation status, WHO grade and histological classification are generally considered to influence glioma prognosis, the present study revealed that patients with IDH1/2-wt glioma, GBM and WHO grade IV all have high MATH levels.

The mutation status of the TTN gene was strongly associated with glioma recurrence. The TTN gene encodes the titin protein, which performs nucleic acid- and protein-binding functions, and is involved in dilated cardiomyopathy and cardiac conduction disease (38). A recent study demonstrated that TTN serves a role in overgrowth-associated signaling pathways in PTEN-wildtype Bannayan-Riley-Ruvalcaba syndrome (39). Although, to the best of our knowledge, no studies have yet investigated the association between TTN and tumors, data from the TCGA database revealed that the TTN gene has a high frequency of mutation in a number of tumors, including lung ( 724 cases), skin (379 cases), uterus (282 cases), stomach (274 cases), colon (264 cases) and breast (291 cases) tumors (https://portal.gdc.cancer.gov).

In the present study, the mutation status of the TP53, PTEN, EGFR and ATRX genes was not associated with glioma recurrence. Although these were identified to be significant in the univariate Cox regression analysis, the multivariate Cox regression analysis revealed no association with glioma recurrence. These results suggest that numerous confounding factors can interfere with Cox regression analyses. For example, a number of studies have demonstrated that TP53 mutations are significantly associated with short survival times in patients with glioma (40-44). However, not all mutations in TP53 result in a loss in gene transcription; patients with TP53 mutant glioma that retain transcriptional activity exhibit longer survival times (45).

Several refinements must be taken into account in future studies. First, the data used to calculate the MATH values were obtained from a single location in tumor tissues deposited in the TCGA database, and an increasing number of studies confirm that the gene mutations found in different locations within various tumor regions are heterogeneous $(46,47)$. Therefore, whether the MATH values found in a single location in a tumor are representative of the state of the whole tumor should be further investigated by multiple locus sequencing. In addition, MATH values do not comprehensively describe glioma ITH, which results from epigenetic regulation and the tumor microenvironment and needs to be analyzed using advanced methods.

In conclusion, the present study quantified ITH according to MATH values and extended the knowledge concerning the association between ITH and glioma recurrence. Additionally, the present study provided a novel MATH-containing nomogram model that accurately predicted glioma RFS probabilities.

\section{Acknowledgements}

The authors would like to thank Professor Xuhui Wang (Department of Neurosurgery, Daping Hospital and Institute Research of Surgery, Army Medical University, Chongqing, China), for his guidance and financial support in the present study.

\section{Funding}

The present study was supported by grants from the National Natural Science Foundation of China (grant no. 81772662), the Chongqing Science and Technology Commission (grant no. cstc2018jcyjA2025), and the National Natural Science Foundation of China (grant no. 81300965). This work was also supported by a grant from the Chongqing Science and Technology Commission (grant no. cstc2017jcyjBX0021).

\section{Availability of data and materials}

The datasets analyzed during the present study are available in the TCGA repository, (https://portal.gdc.cancer.gov).

\section{Authors' contributions}

MX and LY designed the experiments. PW, WY, JZ and JM collected, combined and analyzed the data. PW created the images and tables. LX and ML validated the data. All authors wrote and revised the manuscript.

\section{Ethics approval and consent to participate}

Not applicable.

\section{Patient consent for publication}

Not applicable.

\section{Competing interests}

The authors declare that they have no competing interests.

\section{References}

1. Wood MD, Halfpenny AM and Moore SR: Applications of molecular neuro-oncology-A review of diffuse glioma integrated diagnosis and emerging molecular entities. Diagn Pathol 14: 29, 2019.

2. Freese C, Takiar V, Fouladi M, DeWire M, Breneman J and Pater L: Radiation and subsequent reirradiation outcomes in the treatment of diffuse intrinsic pontine glioma and a systematic review of the reirradiation literature. Pract Radiat Oncol 7: 86-92, 2017. 
3. Lin L, Cai J and Jiang C: Recent advances in targeted therapy for glioma. Curr Med Chem 24: 1365-1381, 2017.

4. Kline C, Felton E, Allen IE, Tahir P and Mueller S: Survival outcomes in pediatric recurrent high-grade glioma: Results of a 20 -year systematic review and meta-analysis. J Neurooncol 137 103-110, 2018.

5. Johnson BE, Mazor T, Hong C, Barnes M, Aihara K, McLean CY, Fouse SD, Yamamoto S, Ueda H, Tatsuno K, et al: Mutational analysis reveals the origin and therapy-driven evolution of recurrent glioma. Science 343: 189-193, 2014.

6. Sottoriva A, Spiteri I, Piccirillo SG, Touloumis A, Collins VP, Marioni JC, Curtis C, Watts C and Tavaré S: Intratumor heterogeneity in human glioblastoma reflects cancer evolutionary dynamics. Proc Natl Acad Sci USA 110: 4009-4014, 2013.

7. Mazor T, Pankov A, Johnson BE, Hong C, Hamilton EG, Bell RJA, Smirnov IV, Reis GF, Phillips JJ, Barnes MJ, et al DNA methylation and somatic mutations converge on the cell cycle and define similar evolutionary histories in brain tumors. Cancer Cell 28: 307-317, 2015.

8. Zhao Y, Alakhova DY and Kabanov AV: Can nanomedicines kill cancer stem cells? Adv Drug Deliv Rev 65: 1763-1783, 2013.

9. Brown TM and Fee E: Rudolf carl virchow: Medical scientist, social reformer, role model. Am J Public Health 96: 2104-2105, 2006.

10. Nowell PC: The clonal evolution of tumor cell populations. Science 194: 23-28, 1976.

11. Kim J, Lee IH, Cho HJ, Park CK, Jung YS, Kim Y, Nam SH, Kim BS, Johnson MD, Kong DS, et al: Spatiotemporal evolution of the primary glioblastoma genome. Cancer Cell 28: 318-328, 2015.

12. Kim H, Zheng S, Amini SS, Virk SM, Mikkelsen T, Brat DJ, Grimsby J, Sougnez C, Muller F, Hu J, et al: Whole-genome and multisector exome sequencing of primary and post-treatment glioblastoma reveals patterns of tumor evolution. Genome Res 25: 316-327, 2015

13. Sidow A and Spies N: Concepts in solid tumor evolution. Trends Genet 31: 208-214, 2015.

14. Ibragimova MK, Tsyganov MM and Litviakov NV: Natural and chemotherapy-Induced clonal evolution of tumors. Biochemistry (Mosc) 82: 413-425, 2017

15. Mroz EA and Rocco JW: MATH, a novel measure of intratumor genetic heterogeneity, is high in poor-outcome classes of head and neck squamous cell carcinoma. Oral Oncol 49: 211-215, 2013

16. Mroz EA, Tward AD, Hammon RJ, Ren Y and Rocco JW: Intra-tumor genetic heterogeneity and mortality in head and neck cancer: Analysis of data from the cancer Genome Atlas. PLoS Med 12: e1001786, 2015.

17. Rajput A, Bocklage T, Greenbaum A, Lee JH and Ness SA Mutant-allele tumor heterogeneity scores correlate with risk of metastases in colon cancer. Clin Colorectal Cancer 16: e165-e170, 2017.

18. Ma D, Jiang YZ, Liu XY, Liu YR and Shao ZM: Clinical and molecular relevance of mutant-allele tumor heterogeneity in breast cancer. Breast Cancer Res Treat 162: 39-48, 2017.

19. Shen S, Wei Y, Zhang R, Du M, Duan W, Yang S, Zhao Y, Christiani DC and Chen F: Mutant-allele fraction heterogeneity is associated with non-small cell lung cancer patient survival. Oncol Lett 15: 795-802, 2018.

20. Turkalp Z, Karamchandani J and Das S: IDH mutation in glioma: New insights and promises for the future. JAMA Neurol 71: $1319-1325,2014$

21. Alexander BM and Cloughesy TF: Adult glioblastoma. J Clin Oncol 35: 2402-2409, 2017.

22. Li S, Yang R, Sun X, Miao S, Lu T, Wang Y, Wo Y and Jiao W: Identification of SPP1 as a promising biomarker to predict clinical outcome of lung adenocarcinoma individuals. Gene 679: 398-404, 2018

23. Claus EB, Walsh KM, Wiencke JK, Molinaro AM, Wiemels JL, Schildkraut JM, Bondy ML, Berger M, Jenkins R and Wrensch M: Survival and low-grade glioma: The emergence of genetic information. Neurosurg Focus 38: E6, 2015

24. Babu R, Bagley JH, Park JG, Friedman AH and Adamson C: Low-grade astrocytomas: The prognostic value of fibrillary, gemistocytic, and protoplasmic tumor histology. J Neurosurg 119: 434-441, 2013

25. Tom MC, Park DYJ, Yang K, Leyrer CM, Wei W, Jia X, Varra V, Yu JS, Chao ST, Balagamwala EH, et al: Malignant transformation of molecularly classified adult low grade glioma. Int J Radiat Oncol Biol Phys: Aug 25, 2019 (Epub ahead of print).

26. Tykocki $\mathrm{T}$ and Eltayeb M: Ten-year survival in glioblastoma. A systematic review. J Clin Neurosci 54: 7-13, 2018.
27. Ghosh D, Nandi S and Bhattacharjee S: Combination therapy to checkmate Glioblastoma: Clinical challenges and advances. Clin Transl Med 7: 33, 2018.

28. Lee JK, Wang J, Sa JK, Ladewig E, Lee HO, Lee IH, Kang HJ, Rosenbloom DS, Camara PG, Liu Z, et al: Spatiotemporal genomic architecture informs precision oncology in glioblastoma. Nat Genet 49: 594-599, 2017.

29. Suzuki H, Aoki K, Chiba K, Sato Y, Shiozawa Y, Shiraishi Y, Shimamura T, Niida A, Motomura K, Ohka F, et al: Mutational landscape and clonal architecture in grade II and III gliomas. Nat Genet 47: 458-468, 2015.

30. Qazi MA, Vora P, Venugopal C, Sidhu SS, Moffat J, Swanton C and Singh SK: Intratumoral heterogeneity: Pathways to treatment resistance and relapse in human glioblastoma. Ann Oncol 28: 1448-1456, 2017.

31. BernstockJD, Mooney JH,Ilyas A, Chagoya G,Estevez-OrdonezD, Ibrahim A and Nakano I: Molecular and cellular intratumoral heterogeneity in primary glioblastoma: Clinical and translational implications. J Neurosurg 1-9, Aug 23, 2019, (Epub ahead of print).

32. Yang MC, Loh JK, Li YY, Huang WS, Chou CH, Cheng JT, Wang YT, Lieu AS, Howng SL, Hong YR and Chou AK: Bcl2L12 with a BH3-like domain in regulating apoptosis and TMZ-induced autophagy: A prospective combination of ABT-737 and TMZ for treating glioma. Int J Oncol 46: 1304-1316, 2015.

33. Rocha CR, Garcia CC, Vieira DB, Quinet A, de Andrade-LimaLC, Munford V, Belizário JE and Menck CF: Glutathione depletion sensitizes cisplatin- and temozolomide-resistant glioma cells in vitro and in vivo. Cell Death Dis 6: e1727, 2015.

34. Turaga SM and Lathia JD: Adhering towards tumorigenicity: Altered adhesion mechanisms in glioblastoma cancer stem cells. CNS Oncol 5: 251-259, 2016

35. Chen JW, Zhou CF and Lin ZX: The influence of different classification standards of age groups on prognosis in high-grade hemispheric glioma patients. J Neurol Sci 356: 148-152, 2015.

36. Gallego Perez-Larraya J and Delattre JY: Management of elderly patients with gliomas. Oncologist 19: 1258-1267, 2014

37. Rasmussen BK, Hansen S, Laursen RJ, Kosteljanetz M, Schultz H, Nørgård BM, Guldberg R and Gradel KO: Epidemiology of glioma: Clinical characteristics, symptoms, and predictors of glioma patients grade I-IV in the the Danish Neuro-Oncology Registry. J Neurooncol 135: 571-579, 2017.

38. Chauveau C, Rowell J and Ferreiro A: A rising titan: TTN review and mutation update. Hum Mutat 35: 1046-1059, 2014.

39. Yehia L, Ni Y and Eng C: Germline TTN variants are enriched in PTEN-wildtype Bannayan-Riley-Ruvalcaba syndrome. NPJ Genom Med 2: 37, 2017

40. Cho SY, Park C, Na D, Han JY, Lee J, Park OK, Zhang C, Sung CO, Moon HE, Kim Y, et al: High prevalence of TP53 mutations is associated with poor survival and an EMT signature in gliosarcoma patients. Exp Mol Med 49: e317, 2017.

41. Todorova PK, Fletcher-Sananikone E, Mukherjee B, Kollipara R, Vemireddy V, Xie XJ, Guida PM, Story MD, Hatanpaa K, Habib AA, et al: Radiation-induced DNA damage cooperates with heterozygosity of TP53 and PTEN to generate high-grade gliomas. Cancer Res 79: 3749-3761, 2019.

42. Pessoa IA, Amorim CK, Ferreira WAS, Sagica F, Brito JR, Othman M, Meyer B, Liehr T and de Oliveira EHC: Detection and correlation of single and concomitant TP53, PTEN, and CDKN2A alterations in gliomas. Int J Mol Sci 20: pii: E2658, 2019.

43. Jesionek-Kupnicka D, Braun M, Trabska-Kluch B, Czech J, Szybka M, Szymańska B, Kulczycka-Wojdala D, Bieńkowski M, Kordek R and Zawlik I: MiR-21, miR-34a, miR-125b, miR-181d and miR-648 levels inversely correlate with MGMT and TP53 expression in primary glioblastoma patients. Arch Med Sci 15: 504-512, 2019.

44. Forte IM, Indovina P, Iannuzzi CA, Cirillo D, Di Marzo D, Barone D, Capone F, Pentimalli F and Giordano A: Targeted therapy based on 553 reactivation reduces both glioblastoma cell growth and resistance to temozolomide. Int J Oncol 54: 2189-2199, 2019.

45. Fischer NW, Prodeus A and Gariepy J: Survival in males with glioma and gastric adenocarcinoma correlates with mutant p53 residual transcriptional activity. JCI Insight 3: pii: 1213642018.

46. Seow P, Wong JHD, A hmad-Annuar A, Mahajan A, Abdullah NA and Ramli N: Quantitative magnetic resonance imaging and radiogenomic biomarkers for glioma characterisation: A systematic review. Br J Radiol 91: 20170930, 2018.

47. Aubry M, de Tayrac M, Etcheverry A, Clavreul A, Saikali S, Menei P and Mosser J: From the core to beyond the margin: A genomic picture of glioblastoma intratumor heterogeneity. Oncotarget 6: 12094-12109, 2015.

This work is licensed under a Creative Commons Attribution-NonCommercial-NoDerivatives 4.0 International (CC BY-NC-ND 4.0) License. 